\title{
UNTARGETED METABOLIC FINGERPRINTING REVEALS IMPACT OF GROWTH STAGE AND LOCATION ON COMPOSITION OF SEA BUCKTHORN (Hippophä̈ rhamnoides) LEAVES
}

\section{Short title: Metabolic Fingerprinting of Sea Buckthorn Leaves}

Raghunath Pariyani $^{\mathrm{a}}$, Maaria Kortesniemi ${ }^{\mathrm{a}}$, Jaana Liimatainen ${ }^{\mathrm{a}, 1}$, Jari Sinkkonen ${ }^{\mathrm{b}}$, Baoru Yang $^{\mathrm{a}^{*}}$

${ }^{\text {a }}$ Food Chemistry and Food Development, Department of Biochemistry, University of Turku, FI20014 Turun yliopisto, Finland

${ }^{\mathrm{b}}$ Instrument Centre, Department of Chemistry, University of Turku, FI-20014 Turun yliopisto, Finland

*Author for correspondence

Professor Baoru Yang

Food Chemistry and Food Development, Department of Biochemistry, University of Turku, FI-20014 Turun yliopisto, Finland

E-mail: baoru.yang@utu.fi

Mobile: +35845273788

Word count: 7169

${ }^{1}$ Present address: Department of Food and Nutrition, P.O. Box 66, FI-00014, University of Helsinki, Finland 


\section{ABSTRACT}

Sea buckthorn (Hippophä rhamnoides) is increasingly cultivated to produce raw materials for food

3 and nutraceuticals. There is little knowledge on composition of sea buckthorn leaves (SBLs) and the key factors influencing the composition. This research aims to unravel the metabolic profile of SBLs and the effects of cultivar, location and stage of growth, and climatic conditions on the metabolic profile of SBLs. Leaves of two sea buckthorn cultivars grown in the south and north of

7 Finland during two consecutive growth seasons were studied using untargeted NMR metabolomics.

8 The highest variance in the metabolic profile was linked to the growth stage, wherein, leaves from the first seven weeks of harvest were characterized with higher abundance of polyphenols, while relatively higher abundance of carbohydrates and sugars was observed in the later weeks. The growth location attributed for the second highest variation, wherein, the north-south comparison identified fatty acids and sugars as discriminatory metabolites, and the potential association of metabolome to natural abiotic stressors was revealed. An inverse correlation between carbohydrate/sugar content as well as fatty acids of higher carbon chain length with the temperature variables was evident. The supervised chemometric models with high sensitivity and specificity classified and predicted the samples based on growth stage and location, and cultivar. Non-targeted NMR-metabolomics revealed the metabolic profile of SBLs and their variation associated with various biotic and abiotic factors. Cultivar and growth stage are key factors to consider when harvesting SBLs for use in food and nutraceuticals.

Keywords: Hippohaë rhamnoides; Sea buckthorn leaves; Growth stage; NMR metabolomics; Weather conditions. 
24 Globally Sea buckthorn cultivation has been rapidly increasing due to the known health-promoting 25 benefits of the berries and leaves of the plant. The current research obtained new comprehensive 26 information on the compositional profile of sea buckthorn leaves as well as the impact of major 27 contributory factors such as cultivars, the advancement of growth stage, geographical location, and 28 weather parameters. The findings of this research provide new knowledge and guidance for plant 29 breeding, cultivation and commercial utilization of sea buckthorn leaves as raw materials for food, 30 feed, and nutraceuticals. 


\section{INTRODUCTION}

Sea buckthorn (Hippophä rhamnoides L. of Elaeagnaceae) is native to Eurasia and currently cultivated in many regions of the world, especially in Europe, Asia and the North America, owing to its broad potential in various applications, especially within food and nutraceutical industry (Fatima et al., 2015; Suryakumar \& Gupta, 2011 ). Traditionally, the sea buckthorn leaves (SBLs) have been used as ethnobotanical medicine and horse feed (Suryakumar \& Gupta, 2011; Singh et al., 2005). From the beginning of this century, aqueous infusions of SBLs have been in use as herbal tea in the Asian and European regions, thus bringing the otherwise under-utilised by-product of the plant to the frontline. The SBLs tea powder has been shown to decrease the levels of murine hepatic triglycerides and cholesterol as well as elevate fecal lipid excretion (Lee et al., 2011). The potential of SBLs extracts in protection against oxidative stress has been demonstrated in various in vitro studies (Cho et al., 2017; Kim et al., 2017). In addition, various pharmacological roles of SBLs, such as, anti-inflammatory (Ganju et al., 2005), anti-cataract (Dubey, Deep \& Singh, 2016), anti-visceral obesity (Lee et al., 2011), anti-tumor (Zhamanbaeva, Murzakhmetova, Tuleukhanov, \& Danilenko 2014), and anti-microbial (Yogendra Kumar, Tirpude, Maheshwari, Bansal, \& Misra, 2013; Michel, Destandau, Le Floch, Lucchesi, \& Elfakir, 2012) are also reported.

SBLs are rich in polyphenols composed of monomeric $C$-glycosidic ellagitannins (ETs) and flavonoids (Tian et al., 2017; Suvanto \& Salminen, 2016; Fatima et al., 2015; Yoshida, Tanaka, Chen, \& Okuda, 1991). The antioxidative capacity of the SBLs tea extract correlates to the polyphenol content (Cho et al., 2014). The highest concentration of total phenolics in the SBLs of Russian origin grown in Sweden are reported to be in the leaves harvested at the end of July (Morgenstern, Ekholm, Scheewe, \& Rumpunen, 2014). The total flavonols content of Romanian SBLs varied between 563-1437 mg rutin equivalents per $100 \mathrm{~g}$ dry weight $(\mathrm{dw})$, depending on the genotype (Pop et al., 2013). The leaves of the Finnish cultivars, 'Tytti' and 'Terhi' are reported to have total phenolics concentration at $6047 \mathrm{mg}$ and $7856 \mathrm{mg}$ per $100 \mathrm{~g}$ fresh weight, respectively, quantified via HPLC analysis (Tian et al., 2017). Drying in moderate temperature $\left(50-60{ }^{\circ} \mathrm{C}\right.$ to 
moisture of 1-3\%) helps retaining a higher concentration of phenolic compounds and thus maintaining a high nutraceutical quality of SBLs (Guan, Cenkowski, \& Hydamaka, 2005). In particular, processing technique emulating the fermentation of green tea is recommended for preparing SBLs tea, by which bifidogenicity as well as inhibitory activity on Clostridium perfringens (in vitro digestion assay) were demonstrated ( $\mathrm{Li}$ et al., 2016). Thus, all these reports suggest that factors such as genotypes, harvesting time, and processing techniques significantly affect the phytochemical profile of SBLs and thus their bioactivity. In addition to the polyphenolics, the SBLs are characterized by high levels of ascorbic acid, tocopherols (including $\alpha$-tocopherol) and carotenoids (including $\beta$-carotene) (Pop et al., 2014; Kanayama et al., 2013).

Globally sea buckthorn is known for the health promoting benefits of the berries. Lately the interest in SBLs is fast growing due to the increasing scientific reports on the bioactivities of leaves and leaf extracts. In comparison to the extensive research published on the composition of sea buckthorn berries, much less is known about the metabolic profiles of SBLs. Increasing the knowledge on the compositional profile of SBLs would promote the utilization of the leaves, which are the currently under-utilized but highly valuable materials. This will support the growth of sustainable agriculture and bioeconomy.

Metabolomics is a highly applicable tool in the phytochemical characterization of plant phenotypes and in understanding the complex networks and relations between the plant, its genetic background and the environmental factors (Kim, Choi, \& Verpoorte, 2011). Nuclear magnetic resonance (NMR) spectroscopy based metabolomics has been applied to study the metabolite profiles of tea/herbal tea e.g. green tea (Lee et al., 2015; Lee et al., 2010) and Java tea (Pariyani, Ismail, Ahmad Azam, Abas, $\&$ Shaari, 2017). No previous research has been reported on the metabolomics investigation of SBLs. In our recent study, we investigated the metabolic profile of the sea buckthorn berries of two Finnish cultivars, 'Tytti' and 'Terhi', from two growth locations located at the south and north of 
81 Finland showing strong impact of the latitude of the growth location and respective weather conditions (Kortesniemi, Sinkkonen, Yang, \& Kallio, 2017).

In this research, with an objective of systematically identifying the comprehensive phytochemical profile and variation in SBLs during the growth season, we employed an untargeted metabolic fingerprinting approach combining ${ }^{1} \mathrm{H}$ NMR spectroscopy with multivariate data analysis. SBLs samples of 'Tytti' and 'Terhi' were collected in two consecutive growth years from two growing regions located in the south and north of Finland. The metabolic profile of the leaves and the pattern of variations were identified. The constructed chemometric models form a useful tool for classifying SBLs samples in the future. Furthermore, our study was aimed to unveil the association between SBLs metabolites and environmental factors, in particular, variations in the global metabolome as a result of complex interaction among the climatic variables in open fields, which has not been systematically studied to date. The research provides new comprehensive information on the SBLs phytochemicals/metabolites (variations) with respect to major contributory factors such as genotypes, geographical and weather parameters, time of harvest, as guidance for plant breeding, cultivation and commercial utilization of sea buckthorn leaves as raw materials for food, feed, and nutraceuticals.

\section{MATERIALS AND METHODS}

\subsection{Samples}

The leaves of two sea buckthorn (Hippophä̈ rhamnoides L. ssp. rhamnoides) female genotypes ('Terhi' and 'Tytti') were collected from two locations in southern (Satava, Turku; latitude $60^{\circ} 23^{\prime}$ $\mathrm{N}$, longitude $22^{\circ} 09^{\prime} \mathrm{E}$, altitude $1 \mathrm{~m}$ ) and northern Finland (Tepasto, Kittilä; 68 $02^{\prime} \mathrm{N}, 2^{\circ} 37^{\prime} \mathrm{E}$, $210 \mathrm{~m})$. An indicative map of the growth locations is shown in Fig. S1. In both locations, the sea buckthorns grew in sandy soil without administration of fertilizers or pest control. In Kittilä, supplementary watering was administered through a drip irrigation system. The leaves were 
harvested weekly from two bushes per cultivar at each location during the summers of 2012 and 2013 (weeks 25-39 in the south and weeks 28-40 in the north; Table S1). The sampling was performed randomly from all sides of the foliage. The collected leaves were immediately cooled for transport and stored at $-80{ }^{\circ} \mathrm{C}$. The leaves were ground with mortar and pestle with the aid of liquid nitrogen. The lyophilised leaf powder was stored at $-80{ }^{\circ} \mathrm{C}$ until extraction.

\subsection{Meteorological data}

The meteorological raw data was provided by the Finnish Meteorological Institute (Helsinki, Finland). The data from weather stations of Turku Artukainen (latitude $60^{\circ} 27^{\prime} \mathrm{N}$, longitude $22^{\circ} 10^{\prime}$ E, altitude $8 \mathrm{~m})$ and Kittilä Pokka $\left(68^{\circ} 10^{\prime} \mathrm{N}, 2^{\circ} 47^{\prime} \mathrm{E}, 275 \mathrm{~m}\right)$ were applied. The overall cumulative values from the start of the growth season until the final harvest (temperature sum, precipitation sum and global radiation sum), and weekly averages of relative humidity as well as sunshine hours were calculated (Table S1) and used as variables in the multivariate data analyses.

\subsection{Chemicals}

Methanol- $d_{4}\left(\mathrm{CD}_{3} \mathrm{OD}, 99.8 \mathrm{D}-\%\right)$ and deuterium oxide $\left(\mathrm{D}_{2} \mathrm{O}, 99.96 \mathrm{D}-\%\right)$ were acquired from VWR International Oy (Espoo, Finland). The 3-(trimethylsilyl)propionic-2,2',3,3'- $d_{4}$ acid sodium salt (TSP, 98 D-\%) was acquired from Sigma-Aldrich (St. Louis, MO).

\subsection{Sample preparation}

The extraction of the samples and subsequent acquisition of the NMR spectra were performed in a randomized manner, during July - September 2014. The lyophilised leaf powder $(50.0 \pm 0.2 \mathrm{mg})$ was extracted with $1.2 \mathrm{~mL}$ deuterated solvent $\left(\mathrm{CD}_{3} \mathrm{OD}: \mathrm{D}_{2} \mathrm{O}, 8: 2 \mathrm{v} / \mathrm{v}\right.$, with $\left.0.02 \% \mathrm{TSP}\right)$ by vortexing for $1 \mathrm{~h}$ and centrifuged at $9,600 \times g$ for $10 \mathrm{~min}$ at $20{ }^{\circ} \mathrm{C}$. The supernatant was separated, and $600 \mu \mathrm{L}$ was taken to NMR analysis. If the extracts were not analysed on the same day, they were stored at $-20{ }^{\circ} \mathrm{C}$ until analysis. 
129

130

131

132

NMR spectra were recorded using a Bruker Avance 500 NMR spectrometer (Bruker BioSpin AG, Fällanden, Switzerland) operating at $500.13 \mathrm{MHz}$ for proton and equipped with a broadband inverse autotune probe (BBI-5mm-Zgrad-ATM). The solvent-suppressed ${ }^{1} \mathrm{H}$ NMR spectra were acquired at $25{ }^{\circ} \mathrm{C}$ using a double presaturation pulse programme (Bruker's pulse program lc1pnf2), with 256 scans, an acquisition time of $3.28 \mathrm{~s}$, a relaxation delay of $6.70 \mathrm{~s}$, and with spectral width of $10 \mathrm{kHz}$ consisting of $64 \mathrm{k}$ data points. The parameters used in acquiring $J$-resolved (JRES) spectra were 16 scans, $1 \mathrm{k}$ data points, 128 increments, $2 \mathrm{~s}$ relaxation delay, and a spectral width of $16 \mathrm{ppm}$ in dimensions. The heteronuclear multiple bond coherence (HMBC) spectra were obtained using 64 scans, $1 \mathrm{k}$ data points, $1.5 \mathrm{~s}$ relaxation delay, $256 \mathrm{t} 1$ increments at spectral width of $13 \mathrm{ppm}$ and 220 ppm in the proton and carbon dimensions, respectively. The heteronuclear single quantum coherence (HSQC) spectra were acquired using 32 scans, $1 \mathrm{k}$ data points, 128 increments, $2 \mathrm{~s}$ relaxation delay, and spectral width of $16 \mathrm{ppm}$ and $165 \mathrm{ppm}$ in the proton and carbon dimensions, respectively.

\subsection{Metabolite identification}

The identities of NMR signals were assigned with the aid of literature, metabolite library of the Chenomx NMR Suite 8.3 Professional (Chenomx Inc., Edmonton, Alberta, Canada) and querying the open access web based metabolite databases such as Human Metabolome Database (HMDB, http://www.hmdb.ca), and Biological Magnetic Resonance data Bank (BMRB, http://www.bmrb.wisc.edu). The metabolite identities were duly confirmed by two-dimensional NMR experiments such as JRES, HSQC and HMBC.

\subsection{Data processing and multivariate data analysis}

All raw ${ }^{1} \mathrm{H}$ NMR spectra were processed individually to correct the phasing, baseline, and shim using Chenomx NMR Suite. All the spectra were referenced to the internal standard (TSP) at 0.00 
ppm. The chemical shift region $0.0-10.0 \mathrm{ppm}$ was then integrated to bins of width $0.001 \mathrm{ppm}$ after total area normalization, using the Chenomx software. This dataset comprising of 10,000 bins was used to correct the misalignments of the spectra using the icoshift algorithm, in MATLAB platform, based on the correlational shifting of spectral intervals (Savorani, Tomasi, \& Engelsen, 2010). The dataset was divided into 45 intervals and then average spectrum twice (average2) was used as the target spectrum to realign the misaligned peaks. The spectral region related to residual water (4.684.88) and the regions lacking signals such as $\delta 0.0-0.6$ and $\delta 9.5-10.0$ were removed from the aligned spectra. The newly constructed aligned binned data with 9,140 variables was then reduced to 457 chemical shift bin regions of $0.02 \mathrm{ppm}$ width.

The generated dataset comprising 192 observations and 457 variables was then used in the multivariate data analysis using SIMCA-P 14.1 (Umetrics, Sartorius Stedim Biotech, Umeå, Sweden). The mean centered and Pareto scaled data was subjected to unsupervised principal component analysis (PCA) and supervised Orthogonal Partial Least Squares-Discriminant Analysis (OPLS-DA). The grouping patterns of the SBLs samples in different chemometric analysis were observed with the aid of score plots, wherein the spectra were represented as individual points along the principal components. The variables (metabolites) contributing to the characteristic grouping of the samples observed in the score plots were visualized using their corresponding loading plots.

The validation as well as the evaluation of the optimal fit of the OPLS-DA models were performed by internal validation methods of 100 permutation test, calculation of explained variation $\left(\mathrm{R}^{2} \mathrm{Y}\right.$ (cum)), predictive ability $\left(\mathrm{Q}^{2} \mathrm{Y}(\mathrm{cum})\right)$, and CV-ANOVA values. In addition, external validation using prediction dataset was also carried out in order to assess the fitness and predictive ability of the generated classification models. This was achieved by randomly dividing the dataset into two; first, contains about two third of the samples of the dataset, known as training set, which is used initially to generate the model and the second known as prediction set consisting of the other one 
third of the samples, aimed to predict the accuracy and evaluate the fitness of the model independently.

\section{RESULTS AND DISCUSSION}

\subsection{Metabolic profile of sea buckthorn leaves}

The characteristic one-dimensional ${ }^{1} \mathrm{H}$ NMR, and two dimensional J-RES and HSQC spectra of the SBLs of genotypes 'Tytti' and 'Terhi' extracted in 4:1 methanol water solvent system are shown in Fig. 1. An untargeted metabolic profiling yielded a total of 20 chemical identities, with possible nutritional and pharmacological relevance, belonging to both primary and secondary metabolite classes. Table 1 lists out tentatively identified metabolites with the respective chemical shift assignments, signal multiplicities and coupling constants.

The fatty acid signals were found to be one of the most predominant peaks in the spectra in the upfield region. The presence of saturated and unsaturated fatty acids was evident by the characteristic signals of terminal methyl ( $\mathrm{t}, \delta 0.88,0.95)$, acyl groups of the hydrocarbon chain ( $\mathrm{m}$, $\delta$ 1.2-1.3) and olefinic protons $(\mathrm{m}, \delta 5.34)$. The presence of linoleic acid and linolenic acid was confirmed from two-dimensional HSQC spectra.

The singlet peaks at $\delta 0.67$ and $\delta 0.68$ were attributed to $\mathrm{H}-18$ of the phytosterols. Together with these, other characteristic signals including $\delta 0.83(\mathrm{H}-27, \mathrm{~d}, J=6.0 \mathrm{~Hz}), \delta 0.84(\mathrm{H}-26, \mathrm{~d}, J=6.4$ $\mathrm{Hz}), \delta 0.88(\mathrm{H}-29, \mathrm{t}, J=6.8 \mathrm{~Hz}), \delta 1.01(\mathrm{H}-19, \mathrm{~s})$, and $\delta 1.02(\mathrm{H}-21, \mathrm{~d}, J=6.8 \mathrm{~Hz})$ hinted at the presence of $\beta$-sitosterol/ $\beta$-stigmasterol. In addition, the connectivity of H-26 and $\mathrm{H}-27$ with $\mathrm{C}-25$, as evident by the HMBC correlation at $\delta_{\mathrm{C}} 29.6$, further supported the identification. The presence of a few more singlets in the $\delta 0.75-0.81$ region suggests the possible presence of other phytosterols. The presence of esterified sterols and trienols is already reported in SBLs (Suryakumar \& Gupta, 2011; Guan et al., 2005). In addition to the fatty acids and phytosterols, some amino acids and organic acids were also detected in the $0.5-3.0$ ppm region of the spectra, as listed in Table 1. 
The mid-region (3.0 to $5.5 \mathrm{ppm}$ ) of the spectra, primarily contributed by the characteristic peaks of carbohydrates and sugars, was heavily congested; the presence of glucose, fructose and sucrose have been confirmed by the characteristic anomeric proton doublets at $\delta 4.53 / 5.14, \delta 4.13$ and $\delta$ $5.40 \mathrm{ppm}$, respectively. Other metabolites identified from this region included myo-inositol, Lglutamic acid and gluconic acid.

The aromatic region (6.0-10.0 ppm) of the spectra showed several signals, however, they were less intense compared to other regions. Gallic acid and ellagic acid were identified from their characteristic singlets at $\delta 7.04$ and $\delta 7.53$, and confirmed by the HSQC and HMBC spectra. The region from $\delta 6.25-6.85$ showed several singlets, which have been attributed to ellagitannins (ETs). Structurally, ETs are characterized with the presence of hexahydroxy diphenoyl (HHDP) unit(s) linked to sugar moiety. The H6 proton of each ring contributes distinct singlets in the $\delta 6.2-6.4$ region, which were duly confirmed from the JRES NMR spectra. The presence of valoneoyl and galloyl groups was suggested by the presence of singlets at 7.11, 7.09, 7.06, 6.85, 6.52 and 6.27 in the JRES NMR spectra. Further, the characteristic correlations of the H6 protons with C7 were evident from the HMBC spectra (Fig. S2). In addition, the peaks in the range of $\delta_{\mathrm{C}} 138$ in HSQC are characteristic of the single bond correlation of H6 with C6. The SBLs of Finnish origin are reported to contain on an average $70-73 \mathrm{mg}$ of ETs per gram dry weight (Suvanto, \& Salminen, 2016). The major ETs present in quantifiable levels in SBLs include hippophaenin A, B and C, castalagin, vescalagin, pedunculagin, casuarinin and stachyurin (Suvanto et al., 2018). All these compounds are characterized with high structural similarities; for example, hippophaenin B - C, castalagin - vescalagin, and casuarinin - stachyurin are epimer pairs. Similarly, the only difference of casuarinin and stachyurin from castalagin and vescalagin is the absence of a $\mathrm{C}-\mathrm{C}$ bond between the $\mathrm{B}$ and $\mathrm{C}$ rings. These close structural similarities coupled with the extensive overlap of the signals hindered the unambiguous individual identity assignment of ETs. 
The doublets $(J=8.5 \mathrm{~Hz})$ at $\delta 6.80$ and $\delta 6.95$ represented $\mathrm{H}^{\prime}$ ' of the flavonol aglycone, which was further confirmed by the $\mathrm{HMBC}$ correlation with $\delta_{\mathrm{C}} 123.0$ and $\delta_{\mathrm{C}} 148.8$. The doublet at $\delta 7.63(J=$ 8.7 Hz) is correlated in HSQC with $\delta_{\mathrm{C}} 126.2$, which represents the correlation of the C6' aglycon. Similarly, the HSQC correlations at $\delta_{\mathrm{C}} 116-118$ represent characteristic shifts of C5'. Doublets with a coupling constant of $2.5 \mathrm{~Hz}$ at $\delta 6.36$ and $\delta 6.55$ further endorse the presence of flavonol glycosides. The polyphenol pattern identified by the NMR metabolic profiling of SBLs is in good agreement with previous report that ETs constitute more than $90 \%$ of the total phenolics in SBLs, and the rest constituted by flavonol glycosides (Tian et al., 2017).

The metabolic profile of SBLs differs significantly from that of berries, which is the most commonly utilized/consumed part of the sea buckthorn plant. The most differentiating metabolic feature is the polyphenolic profile. The polyphenolic profile of SBLs is reported to be constituted mostly by ETs, whereas, the flavonol glycosides composed of the isorhamnetin and quercetin glycosides represent the major phenolic compounds in berries (Tian et al., 2017). According to Fatima et al., 2015, SBLs have several-fold higher levels of phenolics with gallic acid as the predominant phenolic acid. SBLs are characterized by the presence of ellagic, sinapic, and cinnamic acids and rutin, and the absence of myricetin, whereas, the berries are rich in $p$-coumaric acid, myricetin and quercetin but lack rutin (Fatima et al., 2015). Our previous study on the NMR metabolic profile of the berries collected from the cultivars 'Tytti' and 'Terhi' identified unique metabolites such as L-quebrachitol, and ethyl as well as methyl $\beta$-D-glucopyranoside (Kortesniemi et al., 2017), however, they could not be identified from the spectra of leaves of the corresponding cultivars. Other constituents of nutritional and sensory significance, including, malic acid, asparagine and ascorbic acid, and several other primary metabolites were also identified from berries (Liu et al., 2017).

\subsection{Unsupervised chemometric investigation using Principal Component Analysis}


Principal component analysis (PCA) is the most commonly used unsupervised dimensionality reduction tool in metabolomics to investigate the main variance, detect grouping trends and outliers in a dataset. The variations of the dataset are visualized along the principal components (PC), wherein, the first PC constitutes the highest explained variation. The focal strength of PCA is that the intragroup variations and/or larger sources of variability in the dataset are highlighted (Worley \& Powers, 2013).

The PCA model generated from the mean-centered and Pareto scaled data showed excellent goodness of fit $\left(R^{2} X_{\text {(cum) }}=0.93\right)$ and high predictive ability $\left(Q^{2}\right.$ (cum) $\left.=0.86\right)$. The first three PCs together constituted for a total of $69.7 \%$ of the variance involved in the dataset (Table 2). As observed from the score scatter plot (Fig. 2 A and Fig. S3), the most significant variation in the analyzed SBLs samples was brought about by the differences in the stages of growth. The samples representing the early stages of growth (samples collected from the beginning of harvest until first week of August) were clustered on the negative axis of the PC1, against those samples representing the later stages seen on the positive axis of PC1. The PC1 constituted for $30.3 \%$ of the total variance of the dataset. The discriminant grouping according to the growth stage was observed uniformly on both the studied cultivars, irrespective of the geographical location. This is further evident from the clustering of initial seven weeks of samples from northern Finland together, although the onset of growth season in the north was delayed (week 22), when compared with the south (week 18).

The corresponding loadings scatter plot (Fig. 2 B) showed that the main variables responsible for the separation on the first principal component (p[1]) were bins 1.25 and $1.29 \mathrm{ppm}$ (fatty acids), and aromatic region constituted by polyphenols (bin labels 6.0-7.2 ppm) on the initial growth stages. The late growth stages were characterized with the chemical shift bins contributed by the sugars and carbohydrate regions (bin label 3.0-4.0 ppm), which are seen on the positive axis of PC1. However, the $\alpha$-glucose (bin 5.15) identified from the characteristic anomeric protons was found to be higher on the initial stages of growth. 
A trend in the distribution of samples according to the growth region could be seen along PC2; however, a clear discrimination of samples of the north from those of the south was brought together with PC3 (Fig. 2 C). The PC2 and PC3 explain $28 \%$ and $12 \%$ of the total variation, respectively. The chemical shift bins representing the acyl groups of fatty acids (bins 1.25, 1.29 and 2.77) seem to have the predominant contribution towards the samples in the north, against those from the sugars and carbohydrates (bin label 3.0-4.0 ppm) in the south (Fig. 2 D). A growing body of literature show that abiotic stressors such as variations in temperature, light, and salinity as well as drought cause accumulation of lipids, including fatty acids, in the plant tissues (Singer, Zou, \& Weselake, 2016; De Bigault Du Granrut \& Cacas., 2016). Higher abundance of fatty acids indicated by the higher intensities of acyl group signals in the SBLs from north Finland might be elicited by the prevalent unique environmental stressor such as low temperature.

The PCA identified and highlighted that among the various variable conditions possibly contributing to the differed metabolite compositions, growth stage exerts the highest effect, followed by the growth region. A separation based on the cultivars was not evident from the unsupervised analysis, suggesting that the variations in metabolic profiles of the leaves of the selected cultivars 'Tytti' and 'Terhi' are more subtle than the growth stages and geographical origin.

\subsection{Supervised chemometric investigation using Orthogonal Projections to Latent Structures-}

Discriminant Analysis (OPLS-DA)

The dataset was subjected to supervised multivariate data analysis, which is particularly used in building models capable of classifying (future) samples using the available spectral data. This is performed by fitting the samples in the discriminant analysis version of OPLS models (i.e., OPLSDA), which is a highly used tool in the field of metabolomics in recent years. The most significant variables contributing to the discrimination of the two groups under analysis were determined by a three-tier approach. At the first step, variables carrying high weighting in the differentiation of the two groups located at the two tails of the S-plot were identified. As a second tier criterion, among 
the variables identified from the S-plot, only those with a Variable Important in Projection (VIP) $\geq$ 1 were considered as discriminant markers. In addition to these, thirdly, the jack-knife bars of the thus selected variables were sorted from the loading column plots. Thus, the systematic approach of combining information from these three approaches ensured the identification of the discriminant variables of statistical significance by filtering out those variables with shared features between the groups.

The OPLS-DA analyses were performed to classify and predict SBLs samples based on the growth stage and growth location, which were identified to be responsible for $70 \%$ of the total variance involved in the whole dataset from the PCA model. In addition to that, the ability to discriminate the cultivars ('Tytti' and 'Terhi') was also investigated.

The validation of the supervised multivariate models, such as OPLS-DA, is particularly important to eliminate the potential risk of over fitting. The plots generated from the permutation tests (100 on both variables) are shown in Fig. S4. All three models proved to have good validity based on their R2Y- and Q2Y-intercept values being lower than 0.3-0.4 and 0.05, respectively (Eriksson et al., 2013). More detailed validation features including R2Y, Q2Y and CV-ANOVA are presented in Table 2. In addition, an external validation method was employed to estimate how well the generated model will perform when applied to new samples, as described in section 3.3.1.

The Fig. 3- A, C and E showed a clear discrimination of samples of early vs late stages of growth, north vs south growth locations, and cultivars 'Tytti' vs 'Terhi', respectively, by component 1 . The model diagnostics including the fit and robustness of the specific OPLS-DA models are presented in Table 2. It is evident from Fig. $3 \mathrm{~B}$ that the early growth stage was characterized with the presence of bins representing the ETs (bins 7.15, 7.13, 7.11, 7.09, 6.67, and 6.65), flavonol glycosides (bins 6.93 and 6.73), and fatty acids (bins $2.15,1.97,1.25$ and 1.23), hinting at their higher abundance in the initial stages of growth compared to the rest of the weeks in the growth period. It was reported that the levels of flavonols and flavonol glycosides in SBLs of Russian origin grown in Sweden 
decrease as the season advances towards the end of July, while the levels of procyanidins and hydrolysable tannins increase (Morgenstern et al., 2014). It could be suggested from the current findings that the general trend of polyphenolic profile, including the flavonols and ellagitannins, in Finnish SBLs reached their peak by the first week of August in the south, while in the north it remained on the higher side until the end of August during the two years investigated. The bins characteristic of the sugars and carbohydrates on the positive axis of the S-plot suggested their higher abundance in the later growth stages. With regard to the metabolites responsible for the discrimination of SBLs samples between north and south growth location, it is clear from Fig. 3 D that the samples from north were characterized with the presence of bins representing the sugars hinting at their higher abundance. The $\alpha$ - (bin 5.15) and $\beta$ - (bin 4.54) glucose are particularly identified to be the discriminating metabolites in the north. On the other hand, samples from south had higher presence of resonance signals at the aromatic regions, in particular those of ETs (bins $7.07,6.83,6.41$ ). Succinate (bin 2.33) was identified to be another significant discriminating marker in samples from south. Comparing the two cultivars, the higher abundance of fatty acids (bins 1.25 and 1.29) in cultivar 'Terhi' was clearly evident from the Fig. 3 F. Other classes of metabolites found to be present in higher quantities in 'Terhi' included carbohydrates and sugars. On the other hand, the samples from 'Tytti' had higher presence of ETs (bins 7.13, 7.11, 7.05, and 6.27) and flavonol glycosides (bins 6.95, 6.93, 6.83, and 6.79).

\subsubsection{Prediction and external validation of the OPLS-DA models}

The $Y$-predicted score scatter plots of the external validation sets of the OPLS-DA models specific to the discrimination of SBLs based on growth stages, growth locations and cultivars are shown in Fig. 4- A, C and E, respectively. Table 2 shows that the models have demonstrated high correct classification rates ranging from $95-100 \%$ based on the criteria of assigning to the class with nearest $Y$-prediction score values, and $85-94 \%$ with a more stringent class assignment criteria set at a $Y$-prediction score value of 0.65 . 
In addition, all three prediction models (on growth stage, growth location, and cultivars) were able to classify the samples with high accuracy indicated by the high sensitivity and specificity summarized via Receiver Operating Characteristic (ROC) plots in Fig. 4- B, D and F. The ROC plot represents the trade-off between the sensitivity (true positives) and specificity (false positives) on the $\mathrm{Y}$ and $\mathrm{X}$ axis, respectively. The area under the ROC curve (AUC) is an estimate of the accuracy of the binary classification, where a value equal to 1.0 represents the complete separation of the two classes (Alonso, Marsal, \& Julià, 2015).

\subsection{Correlation of the identified discriminatory metabolites with the weather parameters}

A PLS-DA model was used to understand the association of metabolic composition of SBLs with the characteristic weather parameters in the north and south growth locations in Finland. The variables of the dataset comprised the chemical shift bins of the identified discriminatory metabolites, i.e., fatty acids, sugars and carbohydrates, polyphenolics, and the chosen weather parameters (Table S1). It is evident from the weather data that north Finland differs from south in having shorter growth period with a cooler growth environment marked with low- temperature, sunshine hours and radiation. The temperature sum of the growth season in the north is about $40 \%$ lower compared to that of the south, in both growth years. However, there was no large difference in the relative humidity between the north and the south.

The constructed PLS-DA model from the UV scaled data was optimally good as shown by the validation via 100 permutation test (Y-intercepts of $R^{2}$ and $Q^{2} 0.099$ and -0.406 , respectively), as well as $R^{2} Y_{\text {(cum) }}=0.643$ and $Q^{2}$ (cum) $=0.469$, respectively. Fig. S5 presents the biplot obtained from PLS-DA, which is a combination of the score and loading plots represented in a single plane. The most striking observation is that all chemical shift bins representing sugars have clustered along with the northern SBLs samples, situated directly opposite to the weather variables related to higher temperature. This suggested an inverse correlation between the abundance of sugars/carbohydrates in SBLs and the temperature. In addition, sugars showed an inverse correlation with radiation and 
sunshine hours and a direct correlation with relative humidity. The soluble sugar concentrations of the Eucalyptus tereticornis leaves decreased at daily ambient air temperature above $20{ }^{\circ} \mathrm{C}$, whereas, it remained relatively constant in the temperature window of $10-20{ }^{\circ} \mathrm{C}$ (Aspinwall et al., 2016). In another study on the cold acclimation of the tea plants, the total soluble sugar content was found significantly rising during the winter, reaching the peak on the day with the lowest recorded temperature. A general up-regulation of the genes involved in the starch, sucrose and raffinose metabolism, as well as sugar transporters was also observed (Yue et al., 2015). The protective role of sugars against dehydration and freezing caused by various environmental stressors are shown to be mediated by their osmoprotectant and interactive ability with the phospholipid bilayer (Wingler, Stangberg, Saxena, \& Mistry, 2012; Shao, He, Bao, \& Mao, 2009).

The observed pattern of fatty acids suggested that their abundance in SBLs is primarily governed by the developmental cue, as all the characteristic signals were clustered near to the samples representing early growth stages, from both south and north locations. The decline in the levels of fatty acids during the later growth stages could be related to the onset of leaf senescence. Previous research on the leaves of Arabidopsis (Arabidopsis thaliana), Brachypodium distachyon, and switchgrass (Panicum virgatum) reported that the leaf senescence brought about a decline in the levels of fatty acids, culminated at about $80 \%$ reduction at the end of the growth season (Yang \& Ohlrogge, 2009). Chemical shift bins of the acyl moiety (1.25 and 1.23) representing the fatty acid chain length were clustered differently from other fatty acid signals on the quadrant opposite to the temperature, radiation and sunshine. This could be suggestive of the accumulation of fatty acids with increased chain length in response to abiotic stressors. Fatty acid carbon chain length is found to be influenced by factors such as temperature as evident by the increased production of erucic acid, a very long chain fatty acid (C22:1), in rapeseed at low temperature (Singer et al., 2016). The underlying mechanism for the influence of temperature on carbon chain length has not been fully elucidated yet (De Bigault Du Granrut \& Cacas., 2016). 
The correlation analysis of metabolites with the weather variables performed in this study was aimed at deriving a primary insight on how the metabolite pattern in SBLs is affected by real time environmental stressors. However, the study is limited by the inclusion of data from only two growth years, which is inferior in its ability to propose a concrete correlation. Hence, monitoring several years' data or studies in controlled growth environments are recommended to establish more specific correlations.

\section{CONCLUSION}

An untargeted analysis using NMR spectroscopy coupled with various chemometric methods identified a wide range of metabolites in SBLs and revealed the variation in metabolic composition of SBLs in respect to the time of harvest, growth location, and cultivar. Leaves harvested early in the summer contain higher proportions of lipids and potential bioactive components, whereas late harvest yielded leaves with relatively higher stores of carbohydrates and sugars. The leaves of cultivar 'Terhi' had relatively more lipids compared to 'Tytti', although these two cultivars shared a closely similar metabolic profile. The correlation analysis of the SBLs metabolites with the environmental factors revealed that abiotic stress conditions, primarily low temperature, promote the accumulation of fatty acids with higher carbon chain length as well as carbohydrates and sugars in the SBLs.

As a polyphenolics-rich side-stream of the berry crop, the SBLs may hold the potential as raw material for food and nutraceutical industry. Our study demonstrated that the phenolic content is the highest in sea buckthorn leaves collected in the early part of the growth period. This should be taken into consideration when harvesting the sea buckthorn leaves for applications in food, feed and nutraceuticals. To our best knowledge, this is the first metabolomics study of SBLs using nontargeted NMR-metabolomics method. The systematic information on the metabolic characterization of SBLs contributed by this study could guide not only in strategizing the collection and effective utilization of SBLs but also in confirming their authenticity/quality control. 
424 Funding from Tekes - the Finnish Innovation Funding Agency (decision 40055/13 for the project 425 "Finnish-Indian Ingredients for Improving Food Safety and Health - FINDfood") is gratefully 426 acknowledged. The authors wish to thank Hannu Lappalainen and Seppo Lappalainen for 427 maintaining the field experiments, Thomas Josserand for technical assistance, and everyone having 428 taken part in the leaf sampling.

\section{Author Contributions}

430 Conception and design of study: JL, MK and BY. Acquisition of data: JL, RP and MK. Analysis 431 and interpretation of data: RP, MK, BY. Drafting the manuscript: RP. Revising the manuscript 432 critically for important intellectual content: MK, BY, JL, JS.

\section{Conflict of interest}

434 The authors declare no conflicts of interest. 


\section{REFERENCES}

Alonso, A., Marsal, S., \& Julià, A. (2015). Analytical methods in untargeted metabolomics: state of the art in 2015. Frontiers in bioengineering and biotechnology, 3, 23.

Aspinwall, M.J., Drake, J.E., Campany, C., Vårhammar, A., Ghannoum, O., Tissue, D.T., Reich, P.B., \& Tjoelker, M.G. (2016). Convergent acclimation of leaf photosynthesis and respiration to prevailing ambient temperatures under current and warmer climates in Eucalyptus tereticornis. New Phytologist, 212(2), 354-367.

Cho, C.H., Jang, H., Lee, M., Kang, H., Heo, H.J., \& Kim, D.O. (2017). Sea buckthorn (Hippophae rhamnoides L.) leaf extracts protect neuronal PC-12 cells from oxidative stress. Journal of Microbiology and Biotechnology, 27(7), 1257-1265.

Cho, H., Cho, E., Jung, H., Yi, H. C., Lee, B., \& Hwang, K. T. (2014). Antioxidant activities of sea buckthorn leaf tea extracts compared with green tea extracts. Food Science and Biotechnology, 23(4), 1295-1303.

De Bigault Du Granrut, A., \& Cacas, J.L. (2016). How Very-Long-Chain Fatty Acids Could Signal Stressful Conditions in Plants?, Frontiers in plant science, 7, 1490.

Dubey, S., Deep, P., \& Singh, A.K. (2016). Phytochemical characterization and evaluation of anticataract potential of sea buckthorn leaf extract. Veterinary ophthalmology, 19(2), 144-148.

Eriksson, L., Byrne, T., Johansson, E., Trygg, J., \& Vikström, C. (2013). Multi- and megavariate data analysis - basic principles and applications (3rd ed.,). Malmö, Sweden: MKS Umetrics AB.

Fatima, T., Kesari, V., Watt, I., Wishart, D., Todd, J.F., Schroeder, W.R., Paliyath, G., \& Krishna, P. (2015). Metabolite profiling and expression analysis of flavonoid, vitamin $\mathrm{C}$ and tocopherol biosynthesis genes in the antioxidant-rich sea buckthorn (Hippophae rhamnoides L.). Phytochemistry, 118, 181-191. 
458 Ganju, L., Padwad, Y., Singh, R., Karan, D., Chanda, S., Chopra, M.K., Bhatnagar, P., Kashyap, R., 459 \& Sawhney, R.C. (2005). Anti-inflammatory activity of Seabuckthorn (Hippophae rhamnoides)

460

461 leaves. International Immunopharmacology, 5(12), 1675-1684.

Guan, T.T., Cenkowski, S., \& Hydamaka, A. (2005). Effect of drying on the nutraceutical quality of sea buckthorn (Hippophae rhamnoides L. ssp. sinensis) leaves. Journal of food science, 70(9), E514-E518.

Kanayama, Y., Sato, K., Ikeda, H., Tamura, T., Nishiyama, M., \& Kanahama, K. (2013). Seasonal changes in abiotic stress tolerance and concentrations of tocopherol, sugar, and ascorbic acid in sea buckthorn leaves and stems. Scientia Horticulturae, 164, 232-237.

Kim, S.J., Hwang, E., Yi, S.S., Song, K.D., Lee, H.K., Heo, T.H., Park, S.K., Jung, Y.J., \& Jun, H.S. (2017). Sea buckthorn leaf extract inhibits glioma cell growth by reducing reactive oxygen species and promoting apoptosis. Applied Biochemistry and Biotechnology, 182(4), 1663-1674.

Kim, H. K., Choi, Y. H., \& Verpoorte, R. (2011). NMR-based plant metabolomics: Where do we stand, where do we go?. Trends in Biotechnology, 29(6), 267-275.

Kortesniemi, M., Sinkkonen, J., Yang, B., \& Kallio, H. (2017). NMR metabolomics demonstrates phenotypic plasticity of sea buckthorn (Hippophä̈ rhamnoides) berries with respect to growth conditions in Finland and Canada. Food Chemistry, 219, 139-147.

Lee, H.I., Kim, M.S., Lee, K.M., Park, S.K., Seo, K.I., Kim, H.J., Kim, M.J., Choi, M.S., \& Lee, M.K. (2011). Anti-visceral obesity and antioxidant effects of powdered sea buckthorn (Hippophae rhamnoides L.) leaf tea in diet-induced obese mice. Food and chemical toxicology, 49(9), 23702376.

Lee, J. E., Lee, B. J., Chung, J. O., Hwang, J. A., Lee, S. J., Lee, C. H., \& Hong, Y. S. (2010). Geographical and climatic dependencies of green tea (Camellia sinensis) metabolites: a ${ }^{1} \mathrm{H}$ NMRbased metabolomics study. Journal of Agricultural and Food Chemistry, 58(19), 10582-10589. 
Lee, J.E., Lee, B.J., Chung, J.O., Kim, H.N., Kim, E.H., Jung, S., Lee, H., Lee, S.J., \& Hong, Y.S. (2015). Metabolomic unveiling of a diverse range of green tea (Camellia sinensis) metabolites dependent on geography. Food chemistry, 174, 452-459.

Li, G., Zhang, J., Liu, E., Wang, F. H., Qi, S., Xiang, X., \& Du, W. (2016). Effects of different sea buckthorn leaf tea processing technologies on nutrient level and fecal microflora in vitro. Journal of Food \& Nutrition Research, 55(3), 205-213.

Liu, Y., Fan, G., Zhang, J., Zhang, Y., Li, J., Xiong, C., Zhang, Q., Li, X., \& Lai, X. (2017). Metabolic discrimination of sea buckthorn from different Hippophä species by ${ }^{1} \mathrm{H}$ NMR based metabolomics. Scientific Reports, 7(1), 1585.

Michel, T., Destandau, E., Le Floch, G., Lucchesi, M. E., \& Elfakir, C. (2012). Antimicrobial, antioxidant and phytochemical investigations of sea buckthorn (Hippophä̈ rhamnoides L.) leaf, stem, root and seed. Food Chemistry, 131(3), 754-760.

Morgenstern, A., Ekholm, A., Scheewe, P., \& Rumpunen, K. (2014). Changes in content of major phenolic compounds during leaf development of sea buckthorn (Hippophä̈ rhamnoides L.). Agricultural and Food Science, 23, 207-219.

Pariyani, R., Ismail, I. S., Ahmad Azam, A., Abas, F., \& Shaari, K. (2017). Identification of the compositional changes in Orthosiphon stamineus leaves triggered by different drying techniques using ${ }^{1}$ H NMR metabolomics. Journal of the Science of Food and Agriculture, 97(12), 4169-4179.

Pop, R. M., Weesepoel, Y., Socaciu, C., Pintea, A., Vincken, J. P., \& Gruppen, H. (2014). Carotenoid composition of berries and leaves from six romanian sea buckthorn (Hippophae rhamnoides L.) varieties. Food Chemistry, 147, 1-9.

Pop, R.M., Socaciu, C., Pintea, A., Buzoianu, A.D., Sanders, M.G., Gruppen, H., \& Vincken, J.P. (2013). UHPLC/PDA-ESI/MS analysis of the main berry and leaf flavonol glycosides from different Carpathian Hippophaë rhamnoides L. varieties. Phytochemical Analysis, 24(5), 484-492. 
Savorani, F., Tomasi, G., \& Engelsen, S.B. (2010). Icoshift: A versatile tool for the rapid alignment of 1D NMR spectra. Journal of Magnetic Resonance, 202(2), 190-202.

Shao, Y., He, Y., Bao, Y., \& Mao, J. (2009). Near-infrared spectroscopy for classification of oranges and prediction of the sugar content. International Journal of Food Properties, 12(3), 644658.

Singer, S.D., Zou, J., \& Weselake, R.J. (2016). Abiotic factors influence plant storage lipid accumulation and composition. Plant Science, 243, 1-9.

Singh, V., Yang, B., Kallio, H., Bala, M., Sawhney, R.C., Gupta, R.K., Mörsel, J.T., Lu, R., \& Tolkachev, O.N. (2005). Seabuckthorn (Hippophae L.): A Multipurpose Wonder Plant, Vol. II: Biochemistry and Pharmacology. India: Daya Publishing House.

Suryakumar, G., \& Gupta, A. (2011). Medicinal and therapeutic potential of sea buckthorn (Hippophae rhamnoides L.). Journal of Ethnopharmacology, 138(2), 268-278.

Suvanto, J., \& Salminen, J. P. (2016). Quantification of the main ellagitannins of Hippophae rhamnoides leaves. Planta Medica, 81(S 01), S317.

Suvanto, J., Tahtinen, P., Valkamaa, S., Engström, M.T., Karonen, M., \& Salminen, J.P. (2018). Variability in Foliar Ellagitannins of Hippophä rhamnoides L. and Identification of a New Ellagitannin, Hippophaenin C. Journal of Agricultural and Food Chemistry, 66(3), 613-620.

Tian, Y., Liimatainen, J., Alanne, A.L., Lindstedt, A., Liu, P., Sinkkonen, J., Kallio, H., \& Yang, B. (2017). Phenolic compounds extracted by acidic aqueous ethanol from berries and leaves of different berry plants. Food chemistry, 220, 266-281.

Wingler, A., Stangberg, E. J., Saxena, T., \& Mistry, R. (2012). Interactions between temperature and sugars in the regulation of leaf senescence in the perennial herb Arabis alpina L. F. Journal of Integrative Plant Biology, 54(8), 595-605. 
529 Worley, B., \& Powers, R. (2013). Multivariate analysis in metabolomics. Current $530 \quad$ Metabolomics, 1(1), 92-107.

531 Yang, Z., \& Ohlrogge, J.B. (2009). Turnover of fatty acids during natural senescence of 532 Arabidopsis, Brachypodium, and switchgrass and in Arabidopsis $\beta$-oxidation mutants. Plant 533 Physiology, 150(4), 1981-1989.

534 Yogendra Kumar, M. S., Tirpude, R. J., Maheshwari, D. T., Bansal, A., \& Misra, K. (2013). 535 Antioxidant and antimicrobial properties of phenolic rich fraction of sea buckthorn (Hippophae 536 rhamnoides L.) leaves in vitro. Food Chemistry, 141(4), 3443-3450.

537 Yoshida, T., Tanaka, K., Chen, X., \& Okuda, T. (1991). Tannins from Hippophae rhamnoides. 538 Phytochemistry, 30(2), 663-666.

539 Yue, C., Cao, H.L., Wang, L., Zhou, Y.H., Huang, Y.T., Hao, X.Y., Wang, Y.C., Wang, B., Yang, 540 Y.J., \& Wang, X.C. (2015). Effects of cold acclimation on sugar metabolism and sugar-related gene 541 expression in tea plant during the winter season. Plant Molecular Biology, 88 6), 591-608.

542 Zhamanbaeva, G. T., Murzakhmetova, M. K., Tuleukhanov, S. T., \& Danilenko, M. P. (2014). 543 Antitumor activity of ethanol extract from Hippophae rhamnoides L. leaves towards human acute 544 myeloid leukemia cells in vitro. Bulletin of Experimental Biology and Medicine, 158(2), 252-255. 
Table 1. Metabolites identified from the ${ }^{1} \mathrm{H}$ and 2D NMR spectra of SBLs extracts with the signal assignments, chemical shift $\left(\delta_{\mathrm{H}}, \mathrm{ppm}\right)$, multiplicity (s, singlet; d, doublet; t, triplet; dd, doublet of doublets; m, multiplet) and scalar coupling constant $(J, \mathrm{~Hz})$ values

No. Metabolite Assignment

Multiplici

$\delta_{\mathbf{H}}$

ty

(Hz)

$1 \quad$ Phytosterols

$0.67-0.81 \quad \mathrm{~s}$

$\beta$-sitosterol/ $\beta$ -

stigmasterol

$\begin{array}{llll}\text { H18 } & 0.68 & \text { s } & - \\ \text { H27 } & 0.83 & \text { d } & 6.0 \\ \text { H26 } & 0.84 & \text { d } & 6.4 \\ \text { H19 } & 1.01 & \text { s } & - \\ \text { H21 } & 1.02 & \text { d } & 6.8\end{array}$

$2 \quad$ Fatty acids

$\begin{array}{llll}\mathrm{CH}_{3} \text { (acyl group), linoleic }(\omega 6) & 0.88 & \mathrm{t} & 7.0 \\ \mathrm{CH}_{3} \text { (acyl group), } \alpha \text {-linolenic } & 0.95 & \mathrm{t} & 7.5 \\ (\omega 3) & & \mathrm{m} & - \\ -\left(\mathrm{CH}_{2}\right) \text { n- (acyl group) } & 1.28 & \mathrm{~m} & - \\ -\mathrm{OCO}_{-} \mathrm{CH}_{2}-\mathrm{CH}_{2} \text { - (acyl group) } & 1.59 & \mathrm{~m} & - \\ -\mathrm{CH}_{2} \mathrm{CH}=\mathrm{CH} \text { (acyl group), } & 2.05 & & \\ \text { unsaturated fatty acids } & & & - \\ -\mathrm{OCO}_{-} \mathrm{CH}_{2}-(\text { acyl group) } & 2.33 & \mathrm{~m} & \\ & 2.35 & \mathrm{t} & 7.1 \\ & & & -\end{array}$

linolenic and linoleic 
acids

3 Threonine

$\mathrm{CH}_{3}$

1.32

d

6.3

H4

3.62

4.9

$4 \quad$ Alanine

$\beta-\mathrm{CH}_{3}$

1.47

7.5

$\alpha-\mathrm{CH}$

3.64

1.90

2.33

d

$5 \quad$ Acetate

$\mathrm{CH}_{3}$

S

6 Succinate

$\alpha, \beta-\mathrm{CH}_{2}$

S

$7 \quad \gamma$ amino butyric

$\beta-\mathrm{CH}_{2}$

1.89

acid

$\alpha-\mathrm{CH}_{2}$

2.26

7.55

$\gamma-\mathrm{CH}_{2}$

2.98

7.05

$8 \quad$ Choline

$\mathrm{CH}_{3}$

3.16

3.21

9.6

3.59

9.6

4.02

2.3

10 Gluconic acid $\mathrm{CH}_{2}$

3.64

$\mathrm{CH}$

3.77

$\mathrm{m}$

$\mathrm{CH}$

4.12

d

3.7

11 L-glutamic acid

2.05

$\mathrm{m}$

2.34

$\mathrm{m}$

3.74

dd

7.2 ,

4.8

12 Fructose $\mathrm{CH}$

4.13

d

8.3

$13 \quad \beta$-glucose

$\mathrm{C}_{1} \mathrm{H}$

4.53

d

8.0 


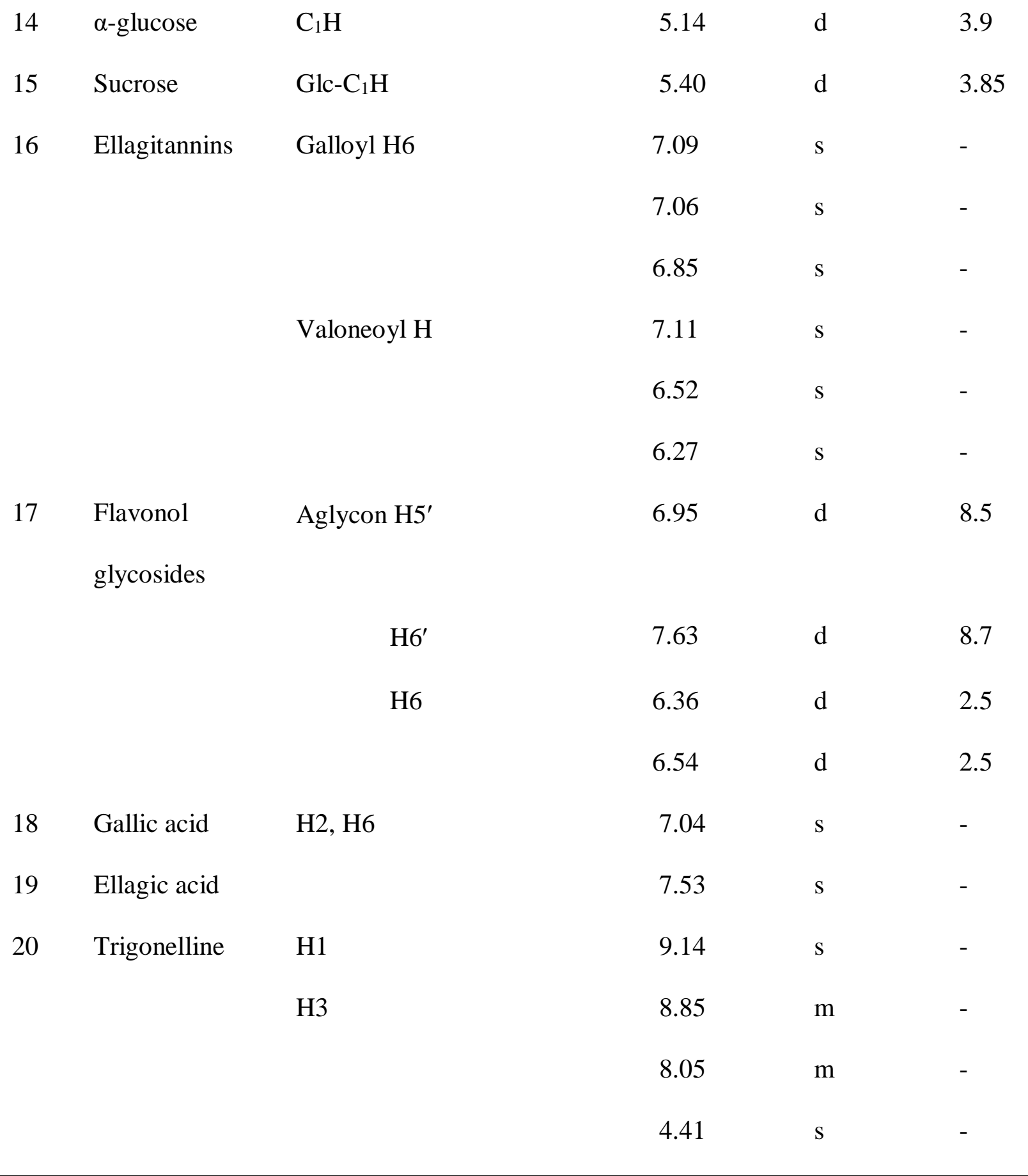


Table 2. Summary of various chemometric models used in the analysis of ${ }^{1} \mathrm{H}$ NMR spectra of SBLs samples

\begin{tabular}{|c|c|c|c|c|c|c|c|c|c|c|}
\hline Parameter of study & $\begin{array}{l}\text { Chemometric } \\
\text { model }\end{array}$ & $\begin{array}{l}\text { Number of } \\
\text { components }\end{array}$ & $\begin{array}{l}R^{2} X \\
\text { (cum) }\end{array}$ & $\begin{array}{l}R^{2} Y \\
\text { (cum) }\end{array}$ & & $\begin{array}{l}\mathrm{CV}- \\
\text { ANOVA }\end{array}$ & \multicolumn{2}{|c|}{ Number of samples } & \multicolumn{2}{|c|}{$\begin{array}{l}\text { External validation } \\
\text { classification rate (\%) }\end{array}$} \\
\hline \multicolumn{11}{|l|}{ General trends and } \\
\hline & PCA & 3 & 0.697 & - & 0.640 & - & - & - & - & - \\
\hline Growth location & OPLS-DA & $1+2+0^{\mathrm{a}}$ & 0.459 & 0.826 & 0.771 & $2.26 \mathrm{e}-037$ & 131 & 61 & 100 & 93.44 \\
\hline Cultivars & OPLS-DA & $1+3+0^{\mathrm{a}}$ & 0.674 & 0.834 & 0.785 & $4.09 \mathrm{e}-037$ & 131 & 61 & 96.72 & 88.52 \\
\hline
\end{tabular}

$546{ }^{\text {a }}$ Predictive component + orthogonal in $X$ component + orthogonal in $Y$ component

$547{ }^{\mathrm{b}}$ Separately chosen samples in random from the main dataset

$548{ }^{\mathrm{c}}$ Class membership assigned based on the proximity of $Y_{\text {pred }}$ values 



Figure 1. NMR spectra of SBLs samples, A: Representative one dimensional ${ }^{1} \mathrm{H}$ NMR spectrum of SBLs collected from south (Sa) and north (Ki) growing regions of Finland, belonging to the cultivars 'Terhi' (Te) and 'Tytti' (Ty), during the summers of 2012 and 2013 , B: Expanded regions of JRES spectrum, C: HSQC spectrum. Refer to Table 1 to interpret the metabolite numbers in B and C. Abbreviations: FA- Fatty acid, GABA- $\gamma$ amino 553 butyric acid. 

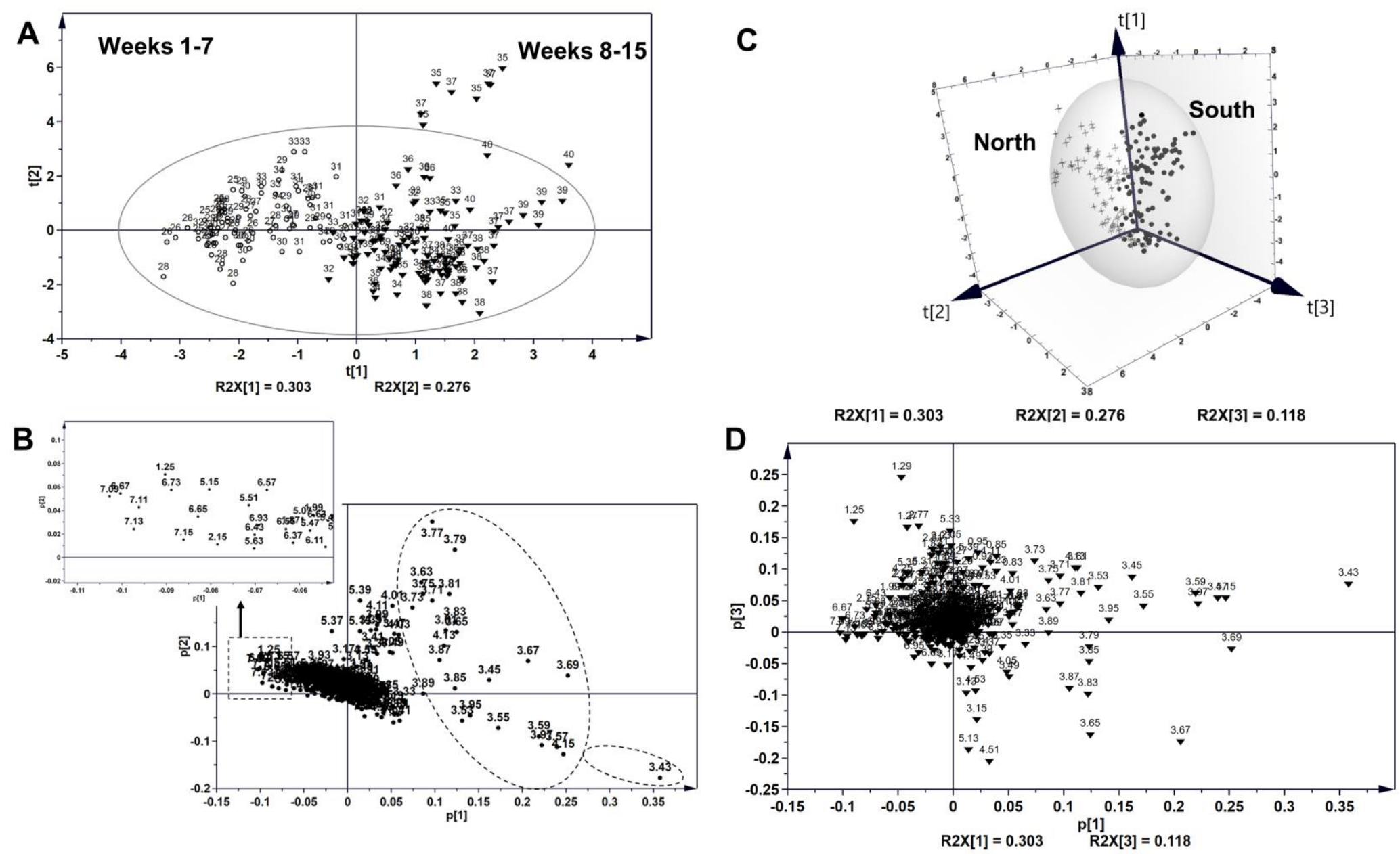

Figure 2. PCA of the binned data generated from the NMR spectra of all the collected samples of SBLs, (192 observations x 457 variables), A: Score scatter plot, B: Loading scatter plot PC1 vs PC2, C: 3D score plot PC1 vs PC2 vs PC3, D: Loading scatter plot of PC1 vs PC3. Symbols of hollow 
A

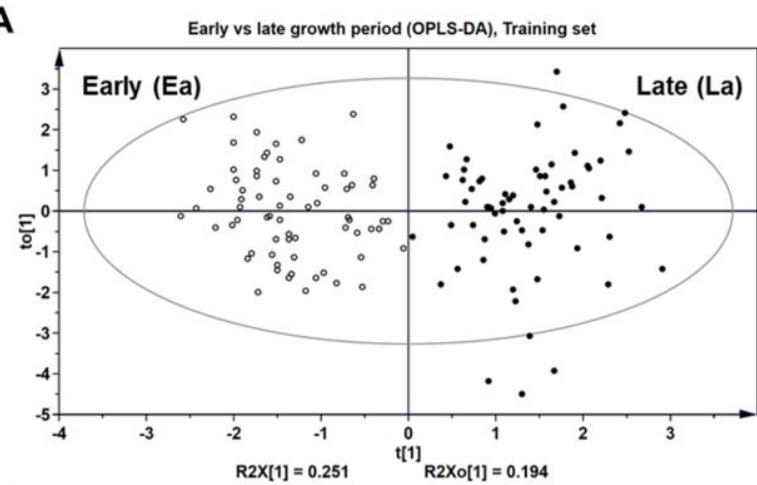

B

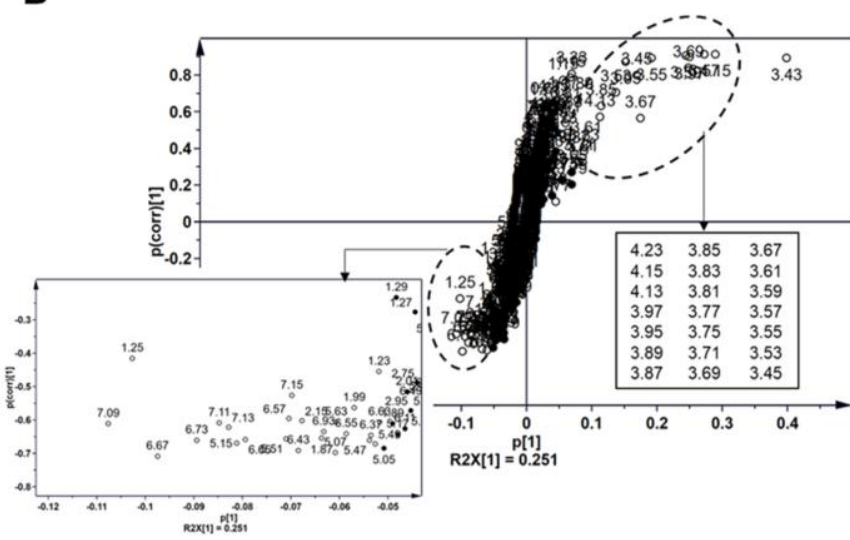

C



D

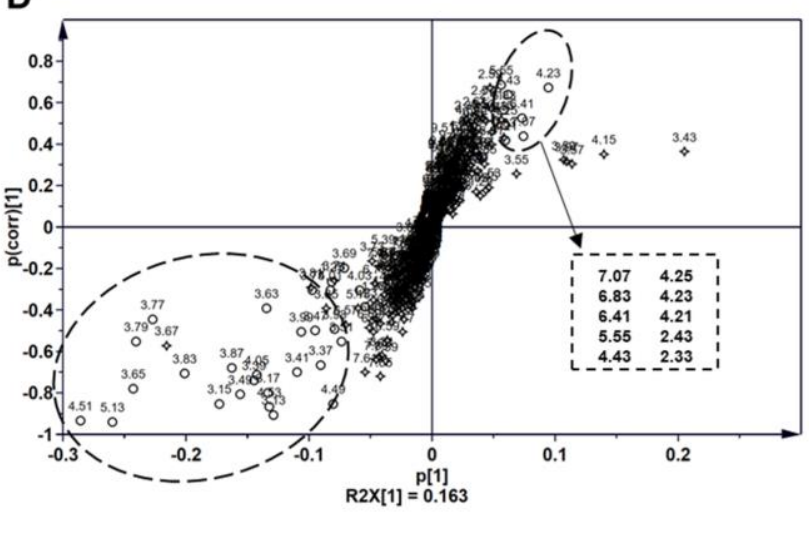

E

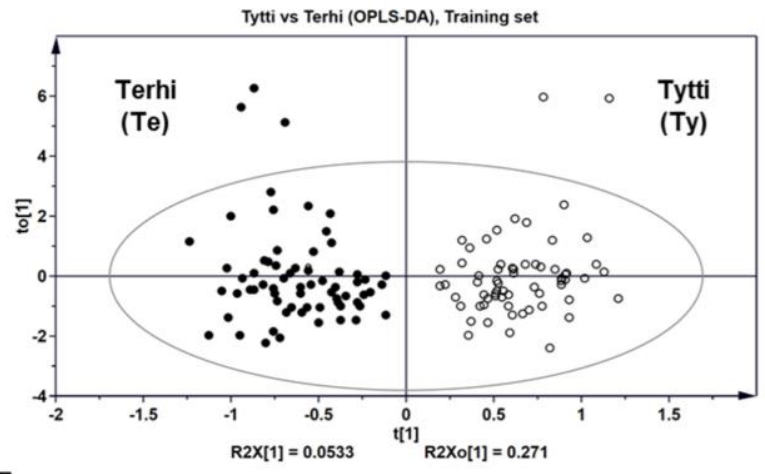

F

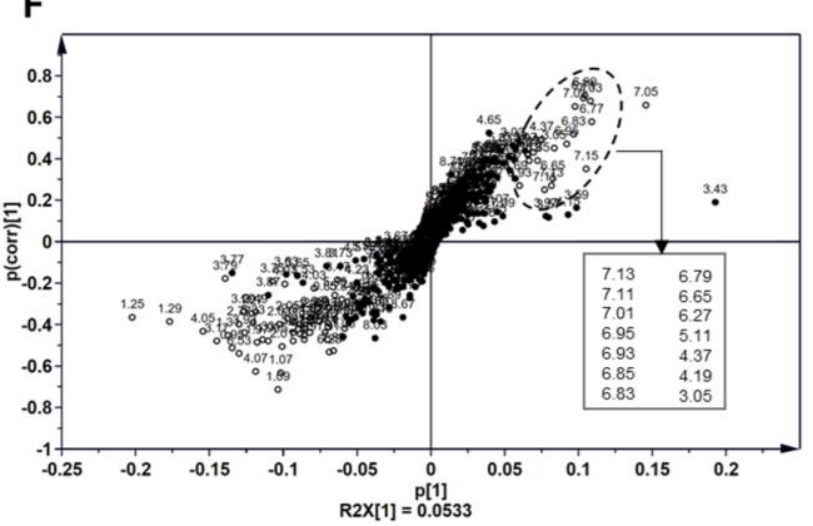

Figure 3. OPLS-DA of the SBLs samples. A, C, and E represent the score scatter plots of training set samples, $t(1)$ vs to(1), of the analysis on growth 
A

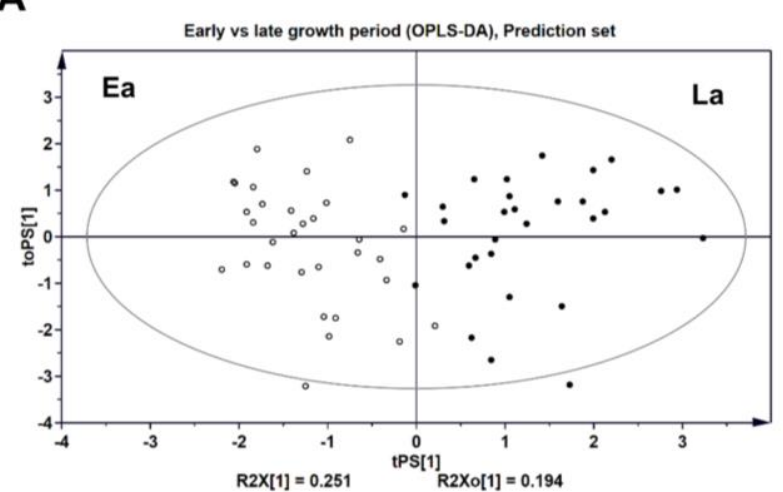

B

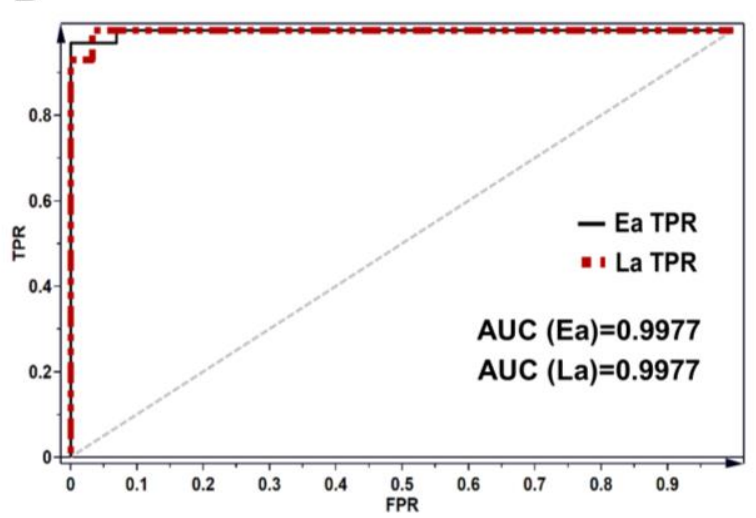

C

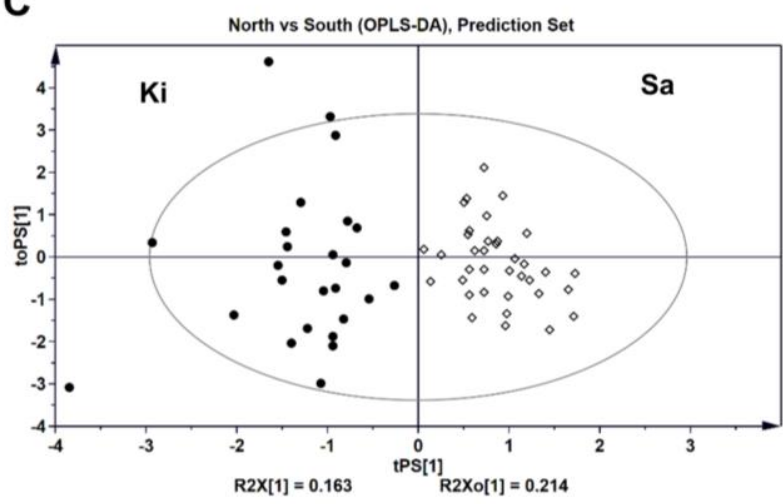

D

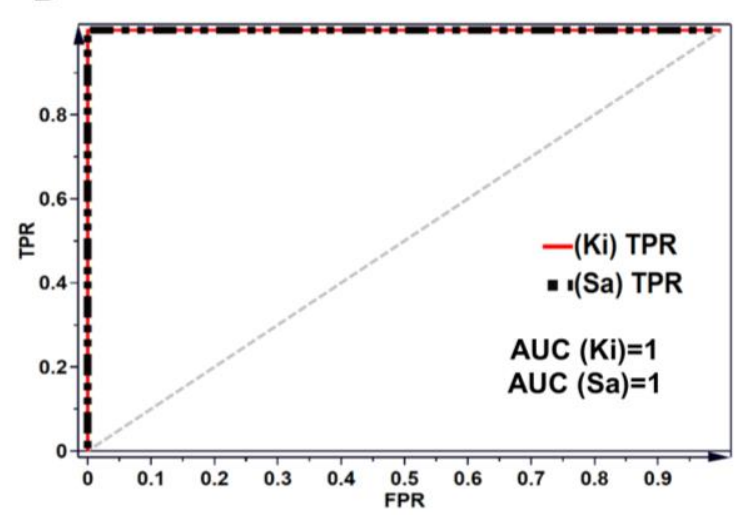

E

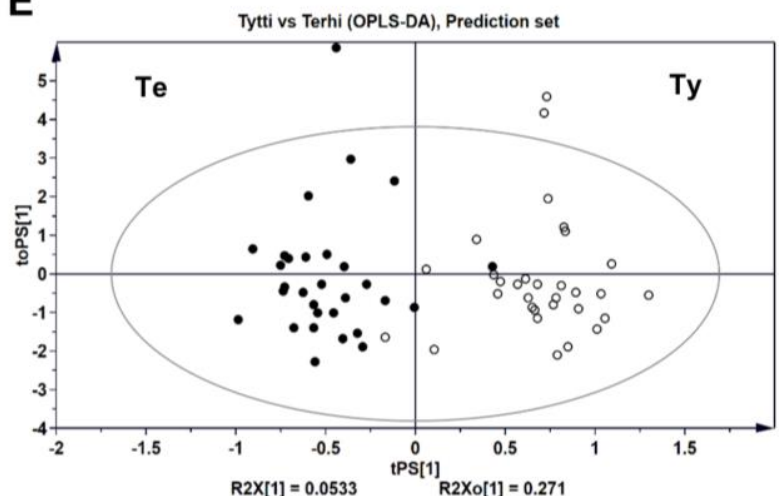

F

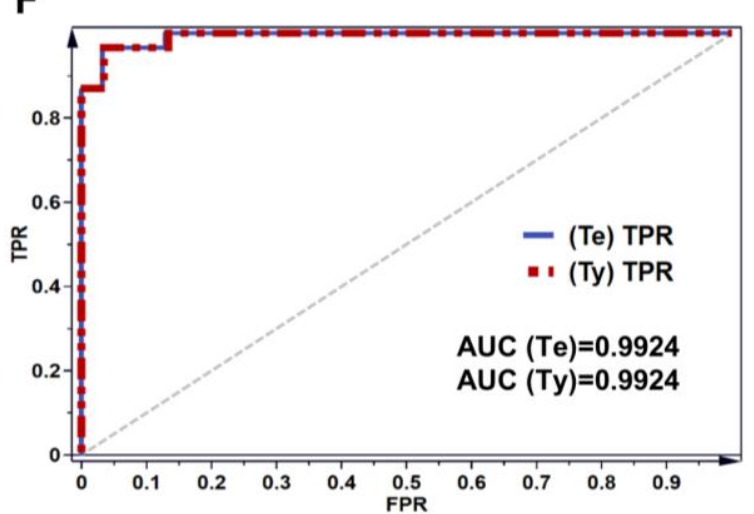

Figure 4. OPLS-DA analysis of the SBLs samples. A, C, and E represent the score scatter plots of prediction set samples, tPS(1) vs toPS(1), of the

D, and F represent the corresponding Receiver Operating Characteristic (ROC) plots of A, C, and E. 